\title{
Access Control and Pilot Allocation for Machine-Type Communications in Crowded Massive MIMO Systems
}

\author{
Ta-Hoang Vo ${ }^{1}$, , Zhi Ding ${ }^{2}$, Quoc-Viet Pham ${ }^{3}$ and Won-Joo Hwang ${ }^{4, *}$ \\ 1 Department of Information and Communication System, Inje University, 197, Inje-ro, Gimhae-si, \\ Gyeongsangnam-do 50834, Korea; tahoanght91@gmail.com \\ 2 Department of Electrical and Computer Engineering, One Shields Avenue, Kemper Hall, \\ University of California, Davis, CA 95616, USA; zding@ucdavis.edu \\ 3 High Safety Core Technology Research Center, Inje University, 197, Inje-ro, Gimhae-si, \\ Gyeongsangnam-do 50834, Korea; vietpq@inje.ac.kr \\ 4 Department of Electronic, Telecommunications, Mechanical Automotive Engineering, Inje University, 197, \\ Inje-ro, Gimhae-si, Gyeongsangnam-do 50834, Korea \\ * Correspondence: ichwang@inje.ac.kr; Tel.: +82-55-320-3847
}

Received: 6 September 2019; Accepted: 4 October 2019; Published: 11 October 2019

\begin{abstract}
Massive machine-type communication (mMTC) in 5G New Radio (5G-NR) or the Internet of Things (IoT) is a network of physical devices such as vehicles, smart meters, sensors, and smart appliances, which can communicate and interact in real time without human intervention. In IoT systems, the number of networked devices is expected to be in the tens of billions, while radio resources remain scarce. To connect the massive number of devices with limited bandwidth, it is crucial to develop new access solutions that can improve resource efficiency and reduce control overhead as well as access delay. The key idea is controlling the number of arrival devices that want to access the system, and then allowing only the strongest device (that has the largest channel gain and each device is able to check whether it is the strongest device) be able to transit to BS. In this paper, we consider a random access problem in massive MIMO context for the collision resolution, in which the access class barring (ACB) factor is dynamically adjusted in each time slot to maximize access success rate for the strongest-user collision resolution (SUCRe) protocol. We propose the dynamic ACB scheme to find optimal ACB factor in the next time slot and then apply SUCRe protocol to achieve a good performance. This method is called dynamic access class barring combined strongest-user collision resolution (DACB-SUCR). In addition, we investigate two different ACB schemes that consist of the fixed ACB and the traffic-aware ACB to compare with the proposed dynamic ACB. Analysis and simulation results demonstrate that, compared with SUCRe protocol, the proposed DACB-SUCR method can remarkably reduce pilot collision, and increase access success rate. It is also shown that the dynamic ACB gives better performance than the fixed ACB and the traffic-aware ACB.
\end{abstract}

Keywords: access class barring; IoT; mMTC; massive MIMO; pilot allocation; access control

\section{Introduction}

Modern telecommunication technologies are developing rapidly with many new applications and methods. Recently, the IoT has become a major driving force in information technology and covers a wide scope of industries and applications. The IoT presents new paradigms such as smart factory, smart home, smart cities and vehicles navigation. In these systems, many autonomous devices communicate with each other without human intervention. 
The main challenges of the IoT are the low-power, the shortage of spectrum resources, and the short range of coverage. Many wireless technologies have already been developed for IoT networks such as ZigBee, SigFox, and Long Range (LoRa) [1-3]. However, network providers prefer to develop technologies that could be compatible with the current cellular networks, e.g., LTE-M, NB-IoT. Moreover, cellular networks can predict and control interference effectively and shall remain useful in dense networks such as an IoT scenario [4]. For these reasons, there are a lot of studies on cellular network to respond to the IoT's needs.

Since the cellular networks have limited bandwidth, new technologies such as massive MIMO transceivers have been proposed to improve the spectral efficiency $[5,6]$. Massive MIMO can easily operate in time-division duplexing (TDD) mode [7], where channel estimation can be more easily available based on the principle of uplink-downlink reciprocity. By using uplink pilots for uplink channel estimation at the massive MIMO transmitter, downlink channel state information (CSI) can be approximately obtained [8]. Nevertheless, in dense MTC device (MTCD) environments, channel estimation faces critical issues that can arise from specific characteristics of mMTC. First, the number of available orthogonal pilot sequences is limited by the dimensionality of the coherence interval of the channel. This leads to a critical shortage of pilots when the number of devices becomes very large. Therefore, the problem of pilot allocation is important in massive MIMO systems [9]. Second, not all of the devices are active simultaneously and frequently. However, each device transmits data to its base station (BS) spontaneously at random, driven by events. Hence, the network should allocate pilots to MTCDs according to a dynamic and adaptive mechanism. One common mechanism is to let each device randomly select one pilot in its uplink transmission to the BS, which however leads to pilot collision [10]. In [11], a belief propagation algorithm is proposed to decontaminate pilot signals and increase system throughput at the cost of access delays. Another interesting protocol called the strongest-user collision resolution (SUCR) has also been proposed this issue, in which the SUCR protocol selects the MTCD with the strongest channel gain as the contention winner in a distributed manner [12]. This protocol can enhance the probability of collision resolution with lower access delay. However, the improvement would be minor in very crowded scenario since the shortage of orthogonal pilot sequences. Various solutions are proposed by 3GPP to alleviate congestions, such as the access class barring (ACB) scheme, slotted access scheme, dynamic allocation of random access (RA) resources, clustering of MTCDs, and back-off scheme. In addition, these also control the random access of MTC devices [13,14]. Among these methods, ACB is one of the most efficient solutions to reduce traffic overload [15-18]. The ACB scheme with the fixed barring factor is proposed in [19]. Using the barring factor as a constant simplifies the system and reduces computation cost. However, it cannot achieve maximum performance. Several papers have proposed other mechanisms to alleviate the congestion caused by MTC communications. In [20], the traffic-aware ACB is proposed to reduce the congestion; however, ACB factor is just adjusted based on a function of the number of arrivals. Unlike the traditional access control approaches, the context-aware access control approaches [21-24] consider a large amount of context information such as location, time and system resources. Therefore, they require that MTC devices have a large CPU capacity and large storage. Nevertheless, we would like to propose an access control protocol based on traditional access control architecture in which even the MTC devices with the moderate CPU capacity and small storage are able to access systems. In this paper, we propose a strategy to improve the access success rate between MTCDs and BS based on SUCRe protocol with access control that is implemented by the ACB scheme. We also propose a new $\mathrm{ACB}$ scheme that is called the dynamic ACB scheme. With the proposed dynamic ACB scheme, BS can adjust the barring factor dynamically after estimating the number of MTCD arrivals in the next time slot, therefore the system performance can be improved efficiently. Moreover, we investigate two different ACB schemes as the fixed ACB scheme and the trafic-aware ACB scheme for SUCRe protocol and RA method to confirm that ACB schemes can improve the performance significantly. A numerical simulation is realized to verify the performance of the proposed DACB-SUCR method. The rest of this paper is organized as follows. System model and preliminaries are presented in Section 2. 
The proposed solutions are discussed and explained in Section 3. In Section 4, we provide simulation results to illustrate the performance of our proposed method as well as the comparison with different existing approaches, and finally conclusions are given in Section 5.

\section{System Model and Preliminaries}

\subsection{System Model}

We consider a single-cell TDD massive MIMO coverage with $N$ single-antenna MTCDs that are served by a BS equipped with $M$ antennas. Such a cellular network with MTCDs is illustrated in Figure 1. Each time slot finds a certain number of active MTCDs, and the remaining MTCDs are inactive. Assume that the MTCDs share $\tau_{p}$ mutually orthogonal pilot sequences, and each sequence is $\tau_{p}$ symbols long. The pilot random access block and data block model is illustrated in Figure 2 . We observe the transmission of MTCDs during one frame $T_{A}$. We call $T_{A}$ the duration of observation. We divide the duration of observation into $I_{A}$ discrete random access slots of the length $\tau_{u}$, where $\tau_{u}$ is not greater than the channel coherence interval. In each time slot, the pilot random access block is followed by a data block. An MTCD uses the pilot random access block to access randomly to BS, and uses data block to transmit its data to BS [25]. After MTCDs send pilots successfully, it can send its data. We only concentrate on the pilot random access process in this paper. During the duration of observation $T_{A}$, the $i$ th time slot is denoted by $\mathrm{RS}_{i}, 1 \leq i \leq I_{A}$. Assume each subframe is transmitted in one time slot. Let $X_{i}$ denote the number of new arrivals during the $\mathrm{RS}_{i}$. According to [13], $X_{i}$ follows the distribution of activation traffic $g(t)$, which is the probability density function of RA requests and the total number of MTCDs $N$. Thus, $X_{i}$ is defined as $X_{i}=N \int_{t_{i-1}}^{t_{i}} g(t) d t, \quad i=1,2, \ldots, I_{A}$.

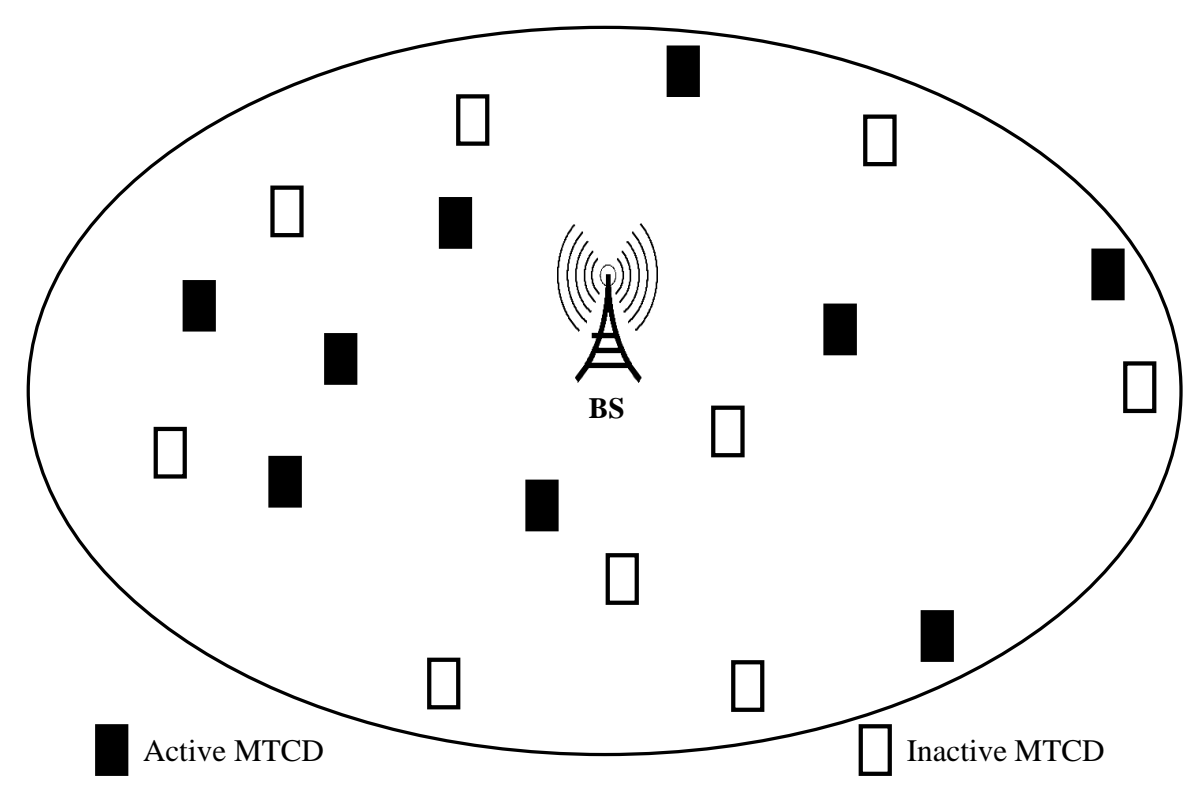

Figure 1. Illustration of cellular network with MTCDs. 


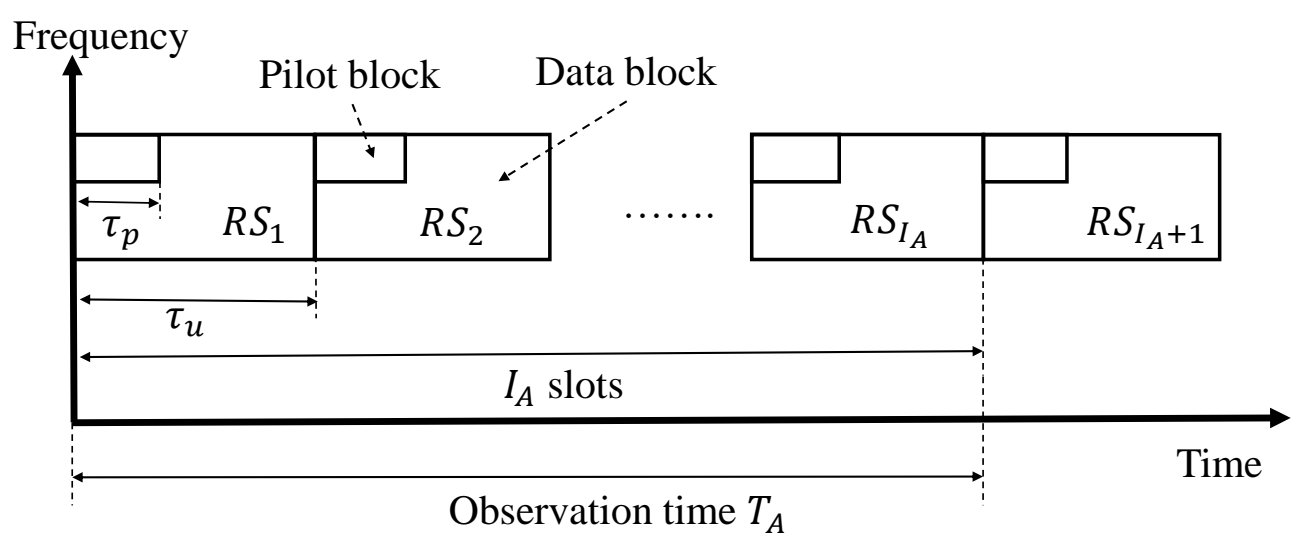

Figure 2. Pilot random access block and data block model.

\subsection{Access Class Barring Scheme}

A well-received method proposed by 3GPP for alleviating the congestion of MTCDs is ACB. When congestion occurs, the ACB scheme is used to prevent the access of MTCDs and reduce overload in the network. In each slot, BS broadcasts an ACB factor and the barring time to all MTCDs. Each MTCD generates a random number $q(0 \leq q \leq 1)$. If $q$ is below the ACB factor, the MTCD passes through the ACB scheme applying the random access procedure. Otherwise, it is barred for a random period of time [26] and has to regenerate the random number. The traditional ACB scheme sets a fixed barring factor during the network access process of MTCDs. A traffic-aware ACB is proposed in [20] to relieve the access delays and increase the access success rate, where the BS changes the barring factor based on the number of device arrivals. In this paper, we propose optimizing the barring factor by maximizing the access success rate. Based on the number of arrivals in the current slot, we compute a barring factor to achieve the maximum access success rate. We then use this barring factor for the next slot.

\subsection{SUCRe Protocol}

SUCRe protocol is presented in detail in [25]. In this paper, we summarize four steps of the SUCRe protocol as follows.

Step 1 Select Random Pilot Sequence: Each MTCD randomly selects a pilot from the set of pilots $P_{0}$ in each RA slot with equal probability $1 / \tau_{p}$, and then this pilot is sent to the BS.

Step 2 Generate and Broadcast Precoded Random Access Response (PRAR), ACB Factor and the Information of Idle Pilots: After the BS receives the pilot signal, it generates the PRAR and broadcast this PRAR to all active MTCDs.

Step 3 Contention Resolution using Pilot Repetition and Pilot Reselection: Each active MTCDs receives the PRAR information and checks whether it has the strongest uplink signal gain, which can be accomplished by comparing its own channel gain and an estimate of the sum of the channel gains of the other contending MTCDs. Only the strongest MTCD should repeat the RA pilot.

Step 4 The BS Allocates Dedicated Payload Pilots: The repeated RA pilots are transmitted by MTCDs to the BS during step 3. Then, the BS checks whether an MTCD suffers from pilot collision or not based on the repeated RA pilot signals. The BS allocates the dedicate data pilots to MTCDS who do not suffers from collision.

In Step 4, the resource allocation in the DL is similar to the precoded response in Step 2. This random access procedure is repeated after a given interval. The MTCDs that do not receive the Dedicated Payload Pilots during Step 4 will choose an integer value as a random waiting time. After the random waiting time, those failed MTCDs will reattempt the random access in the new RA slot. 


\section{Dynamic Access Control and Pilot Allocation Algorithm}

In this section, we use ACB scheme for access control and use SUCRe protocol for pilot allocation. At first, we formulate an optimization problem to maximize the access success rate. In massive MTC systems, the random access of a huge number of MTCDs would not be affordable; therefore, the resource cost should be considered. Here, the access success rate is defined as the ratio between the number of MTCDs that successfully access the BS and the total number of MTCDs that contend to become active in an observation time.

\subsection{Optimization Problem and Algorithm}

The proposed DACB-SUCR method is shown in Figure 3, it consists of access control by the ACB scheme and pilot allocation by SUCRe protocol. Let $P_{0}$ denote the set of mutually orthogonal pilots, $P_{0}=\left\{\xi_{1}, \xi_{2}, \xi_{3}, \ldots, \xi_{\tau_{p}}\right\}$. Moreover, we have $\tau_{p} \ll N$ because of coherence limitation. We assume that each active MTCD chooses a pilot of the $\tau_{p}$ pilot sequences at random in each time slot. When MTCD $k$ attempts to access, it transmits a pilot with a non-zero power $\rho_{k}>0$, otherwise $\rho_{k}=0$. Let $\mathbf{h}_{k} \in \mathbb{C}^{M}$ denote the channel vector between MTCD $k$ and the BS. Assume that the propagation model has channels satisfying two conditions:

$$
\begin{gathered}
\lim _{M \rightarrow \infty} \frac{\left\|\mathbf{h}_{\mathbf{k}}\right\|^{2}}{M}=\beta_{k}, \quad \forall k, \\
\lim _{M \rightarrow \infty} \frac{\mathbf{h}_{k}^{H} \mathbf{h}_{i}}{M}=0, \quad \forall k, i, k \neq i,
\end{gathered}
$$

where $\beta_{k}$ is the strictly positive and is known to MTCD $k$. The study in [27] showed that such channels offer channel hardening and asymptotic favorable propagation. The BS can know the number of pilot collisions and successes, but it cannot know the number of MTCDs that are requesting pilots. To realize the dynamic access control mechanism, the BS needs to estimate the number of MTCDs which are contending for pilots. We present a dynamic access control algorithm by using the estimation mechanism in order to find the optimal $\mathrm{ACB}$ factor $p_{\mathrm{ACB}, i+1}$ for the next time slot. According to [28], the number of active MTCDs is determined by three factors: the number of new arrivals $X_{i}$ in the $i$ th time slot; the number of MTCDs backlogged by the pilot random access failures, which need to re-access the network in $i$ th time slot, denoted as $Y_{i}$; and the number of MTCDs backlogged by the ACB scheme in some previous slots, denoted as $Z_{i}$. Let $K_{i}$ denote the number of active MTCDs during $i$ th slot. Thus, $K_{i}=X_{i}+Y_{i}+Z_{i}$. Let $K_{C, i}$ denote the number of MTCDs passes through the ACB scheme. During the $i$ th time slot, there are $K_{C, i}$ MTCDs applying for pilot allocation by SUCRe protocol after ACB scheme and in each time slot the number of available pilots is $\tau_{p}$. The proposed method consists of two phases: the Estimation phase and Access control phase. 


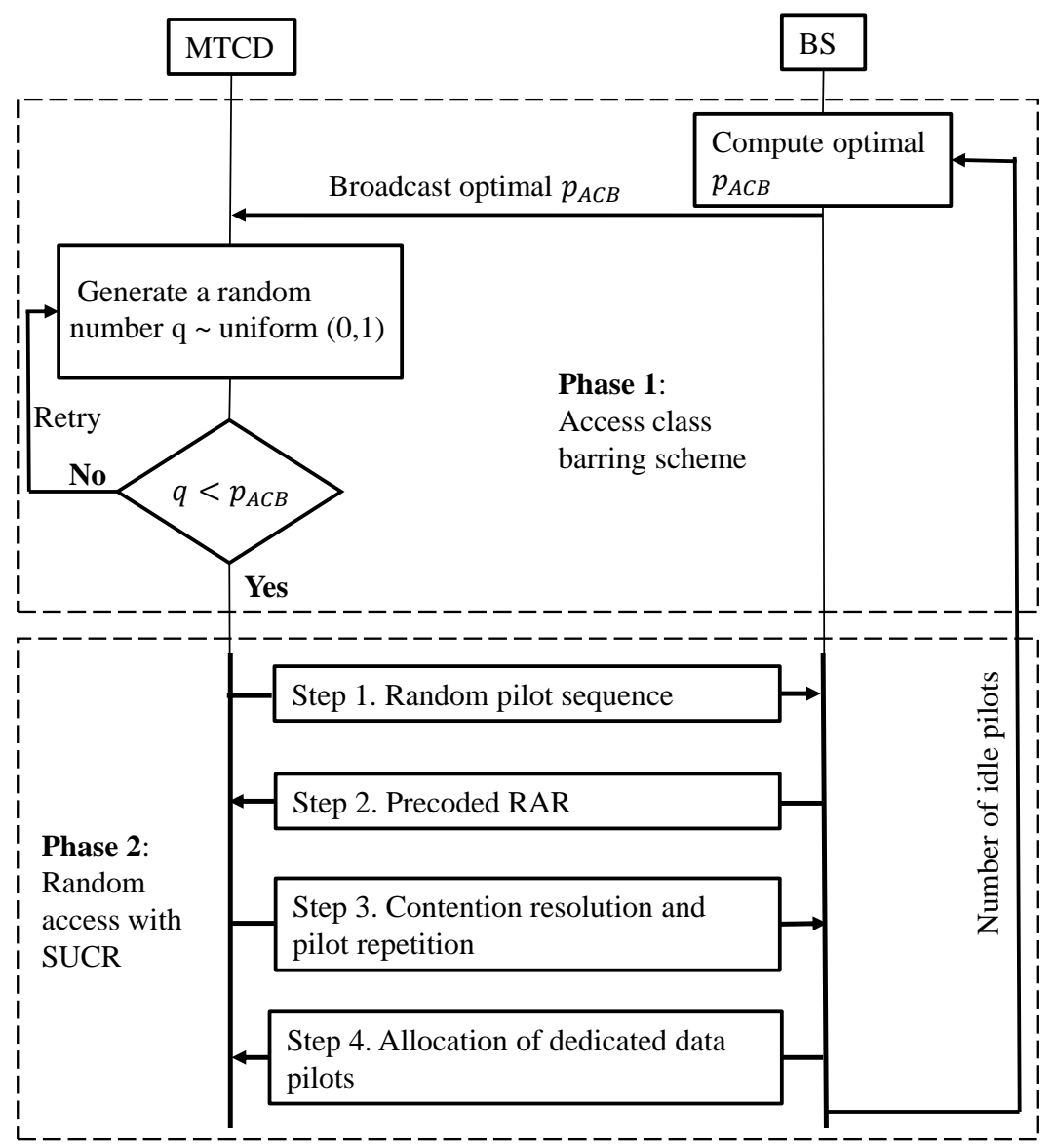

Figure 3. Random access procedure of the strongest-user collision resolution (SUCRe) with dynamic access class barring (ACB).

\subsubsection{Estimation Phase}

Idle pilots can be identified by detecting the signal of pilots. A BS determine the number of idle pilots $\tilde{S}_{i d l e, i}$ in the $i$ th time slot among the total number of available pilots $\tau_{p}$. The probability of the pilots can be computed as

$$
\tilde{p}_{i d l e, i}=\frac{\tilde{S}_{i d l e, i}}{\tau_{p}} .
$$

Moreover, we also compute the probability of the pilots being idle by $p_{i d l e, i}=\left(1-\frac{1}{\tau_{p}}\right)^{K_{C, i}}$. Let $\tilde{p}_{i d l e, i}=p_{i d l e, i}$, the number of contending MTCDs $\hat{K}_{C, i}$ in $i$ th time slot can be estimated as

$$
\hat{K}_{C, i}=\frac{\log \left(\frac{\tilde{S}_{\text {idle }, i}}{\tau_{p}}\right)}{\log \left(1-\frac{1}{\tau_{p}}\right)} .
$$

From $\hat{K}_{C, i}$ and the barring factor $p_{\mathrm{ACB}, i}$ in $i$ th slot, the number of MTCDs in $(i+1)$ th time slot can be estimated by

$$
\hat{K}_{i+1}=\frac{\hat{K}_{C, i}}{p_{\mathrm{ACB}, i}} .
$$




\subsubsection{Access Control Phase}

Since the number of active MTCDs $K_{i}$ can be estimated, the number of MTCDs that pass the ACB scheme is $K_{C, i}=p_{\mathrm{ACB}, i} K_{i}$. Given $K_{C, i}$ contenders, the probability of having $C$ colliding MTCDs that choose the same pilot sequence

$$
p\left(C \mid K_{C, i}\right)=\left(\begin{array}{c}
K_{C, i} \\
C
\end{array}\right)\left(\frac{1}{\tau_{p}}\right)^{C}\left(1-\frac{1}{\tau_{p}}\right)^{K_{C, i}-C},
$$

where $C$ has a binomial distribution with $C \sim B\left(K_{C, i}, \frac{1}{\tau_{p}}\right)$. The probability of having $C$ MTCDs contending to select a pilot sequence, and only one strongest MTCDs repeats this pilot in step 3 is defined by $P\left(D_{C}^{1}\right)$, which can be found in ([25], Equation (20)). Then, we can compute the number of successful access MTCDs $K_{i}^{s}$ during $i$ th time slot as follows: $K_{i}^{s}=\tau_{p} \sum_{C=1}^{K_{C, i}} P\left(D_{C}^{1}\right) P\left(C \mid K_{C, i}\right)$. Let $R_{\text {eff }, i}$ be the access success rate during $i$ th slot. We have

$$
R_{\mathrm{eff}, i}=\frac{K_{i}^{s}}{K_{i}}, \quad i=1,2, \ldots, I_{A}
$$

To maximize the access success rate $R_{\mathrm{eff}}$, the optimum $p_{\mathrm{ACB}, i}^{*}$ can be found via

$$
p_{\mathrm{ACB}, i}^{*}=\underset{0 \leq p_{\mathrm{ACB}, i} \leq 1}{\operatorname{argmax}} R_{\mathrm{eff}, i} \quad i=1,2, \ldots, I_{A} .
$$

The optimization problem in (4) can be solved by the exhaustive search method. This approach limits the number of contending MTCDs for maximizing the access success rate under overload conditions. The dynamic access control and pilot allocation algorithm is summarized in Algorithm 1. Finally, the average access success rate during the observation time $T_{A}$ can be determined as follows

$$
R_{\mathrm{eff}}=\sum_{i=1}^{I_{A}} R_{\mathrm{eff}, i}, \quad i=1,2, \ldots, I_{A}
$$

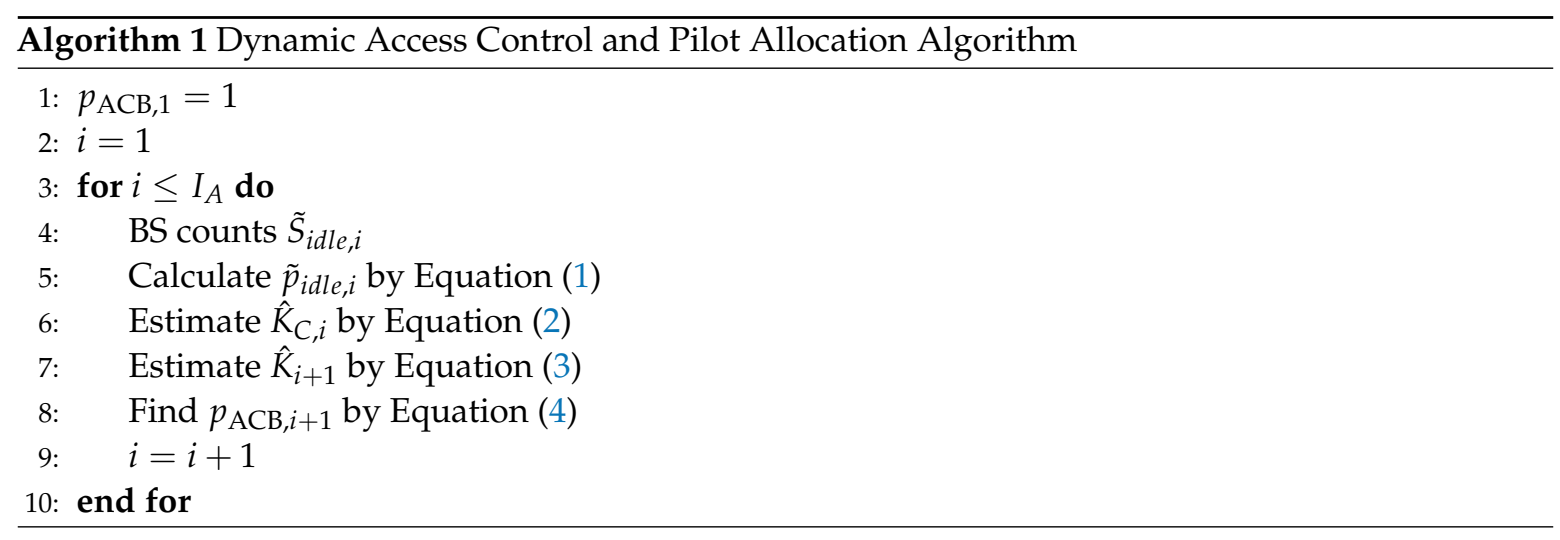

\section{Simulation Results}

We implement Monte-Carlo simulations to compare the performance of the proposed method with several benchmark schemes. In the simulations, the radius of cell is $250 \mathrm{~m}$, the MTCDs are uniformly located in the cell with distance of $25 \mathrm{~m}$ from BS. In the simulation, the urban micro scenario in [29] is used to model the pathloss of the uncorrelated Rayleigh fading with pathloss exponent 3.8. Consider a crowded scenario with $\tau_{p}=50$ during $I_{A}=100$ slots. To illustrate the efficiency of our proposed DACB-SUCR method, we consider comparisons with the following approaches: 
- SUCRe: This method was proposed in [12]. It can resolve pilot collisions when devices select pilot sequence at random to access the network. Each device with the strongest channel gain will be assigned one pilot.

- $\quad \mathrm{RA} w / \mathrm{o} A C B$ : This is a traditional random access procedure without controlling the number of arrival devices. In an access attempt, if more than one device selects the same pilot sequence, they will fail to access the network.

- $\quad$ SUCRe with fixed ACB: We use a fixed ACB factor $\left(p_{A C B}=0.5\right)$ to limit the number of arrival devices and apply the SUCRe protocol for pilot allocation.

- $\quad$ RA with fixed ACB: Similar to SUCRe with fixed ACB, an access control using a fixed ACB factor $\left(p_{A C B}=0.5\right)$ is applied.

- SUCRe with traffic-aware ACB: access control is realized based on traffic-aware ACB factor before the SUCRe protocol will make pilot allocation .

- RA with traffic-aware ACB: random access procedure based on controlling the number of new arrivals by traffic-aware ACB scheme.

Figure 4 shows the variance of the access success rate when the total number of active MTCDs increases. In this simulation, the number of antennas is set as $M=100$. It shows that the access success rate improvement achieved by the proposed DACB-SUCR is remarkably higher than others. At first, when the number of MTCDs is small, most of MTCDs can access the network successfully with all methods. Clearly, the access success rate drops as the number of total MTCDs increases. However, the access success rate of proposed DACB-SUCR is still higher than others. That is because the number of access devices is controlled efficiently by dynamically adjusting the ACB factor. We find that two schemes, when the total number of MTCDs is 10,000, SUCRe with traffic-aware ACB and proposed DACB-SUCR, can handle up to $45 \%$ of the MTCDs. While others just allow less than $25 \%$ of the MTCDs to be accessed successfully.

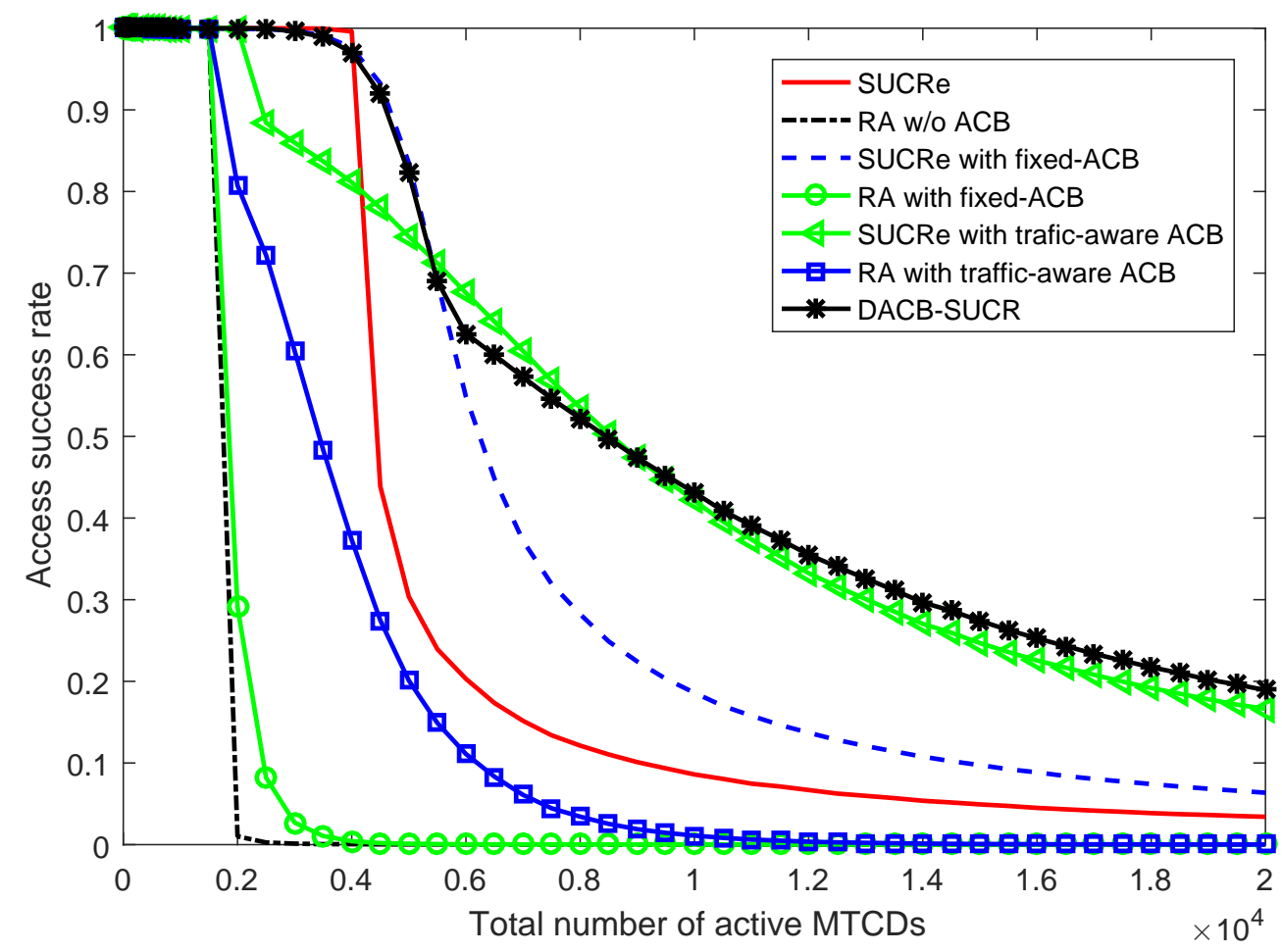

Figure 4. Access success rate.

Figure 5 shows the variance of the access success rate when $M$ increases. The total number of MTCDs is set as $N=10,000$. The access success rate is small at $M=1$, but increases significantly 
when the number of BS antennas gets larger. However, the system performance saturates for $M \geq 15$. Since the number of pilot sequences is finite, the access success rate reaches the saturation even if the number of BS antennas is greater than 30. The increasing number of BS antennas cannot enhance the success access rate indefinitely. Therefore, we need to consider the reasonable number of BS antennas corresponding to the available number of pilot sequences.

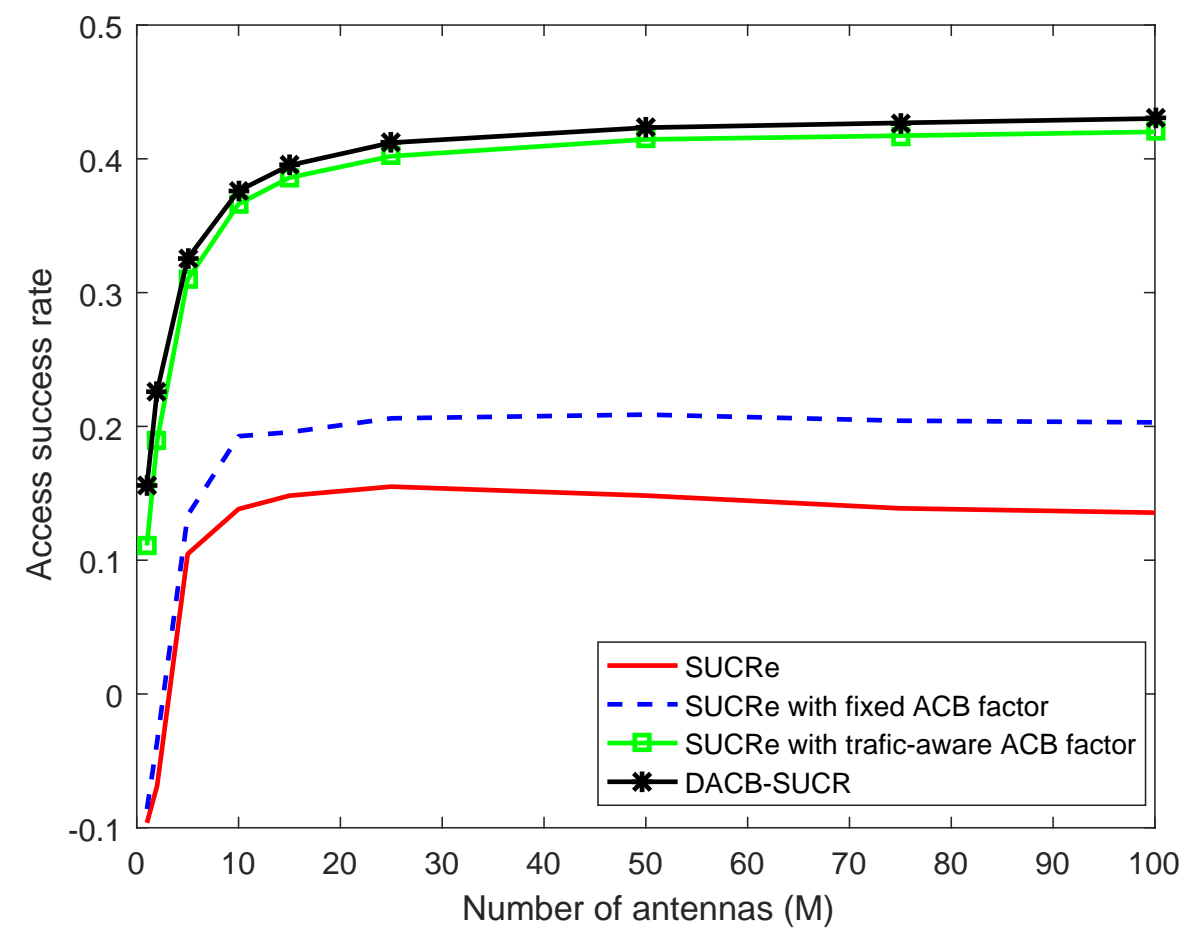

Figure 5. Access success rate for varying number of antennas.

Through Table 1, we can obviously see that the SUCRe with any ACB schemes bring higher performances as compared to the original SUCRe scheme. Among four investigated methods, the proposed DACB-SUCR achieve the highest access success rate. When increasing the number of BS antennas from 5 to 100, SUCRe's performance only increases by around $5 \%$, while the proposed DACB-SUCR's performance increases by more than $10 \%$. Obviously, the proposed DACB-SUCR brings the highest efficiency among these methods.

Table 1. Performance comparison among methods $(N=10,000)$.

\begin{tabular}{lcc}
\hline Method & \multicolumn{2}{c}{ Success } \\
& $\mathbf{M}=\mathbf{5}$ & $\mathbf{M}=\mathbf{1 0 0}$ \\
\hline SUCRe & 10.13 & 14.93 \\
SUCRe with fixed ACB factor & 13.35 & 20.27 \\
SUCRe with traffic-aware ACB factor & 30.67 & 42.07 \\
DACB-SUCR & 32.49 & 43.03 \\
\hline
\end{tabular}

\section{Conclusions}

In this paper, we proposed a new method which exploited ACB scheme and SUCRe protocol to resolve the collision efficiently in crowded massive MIMO network cell. In our work, we also proposed a dynamic ACB scheme that allows BS to choose the optimal ACB factor in the each time slot. Through simulation results, it is shown that the DACB-SUCR method can enhance the access success rate and reduce pilot collisions efficiently and the DACB-SUCR method outperforms the conventional SUCRe protocol. Moreover, compared to the traditional ACB scheme and traffic-aware 
ACB scheme, the proposed dynamic ACB scheme improves the access success rate of both conventional RA procedure and SUCRe protocol.

Author Contributions: Conceptualization, Z.D. and W.-J.H.; Data curation, T.-H.V.; Formal analysis, T.-H.V.; Funding acquisition, W.-J.H.; Investigation, T.-H.V. and W.-J.H.; Methodology, T.-H.V., Z.D., Q.-V.P. and W.-J.H.; Project administration,W.-J.H.; Software, T.-H.V.; Supervision, Z.D. and W.-J.H.; Validation, all the authors; Visualization, T.-H.V.; Writing—original draft, T.-H.V.; Writing—review and editing, all the authors.

Funding: This research was supported by the MSIT(Ministry of Science and ICT), Korea, under the ICT Consilience Creative program (IITP-2019-2016-0-00318) supervised by the IITP(Institute for Information \& communications Technology Planning \& Evaluation).

Conflicts of Interest: The authors declare no conflict of interest.

\section{References}

1. Tomtsis, D.; Kokkonis, G.; Kontogiannis, S. Evaluating existing wireless technologies for IoT data transferring. In Proceedings of the 2017 South Eastern European Design Automation, Computer Engineering, Computer Networks and Social Media Conference, Kastoria, Greece, 23-25 September 2017; pp. 1-4. [CrossRef]

2. Alreshidi, A.; Ahmad, A. Architecting Software for the Internet of Thing Based Systems. Future Internet 2019, 11, 153. [CrossRef]

3. Payne, E.K.; Lu, S.W.Q.W.L. Concept of Designing Thermal Condition Monitoring System with ZigBee/GSM Communication Link for Distributed Energy Resources Network in Rural and Remote Applications. Processes 2019, 7, 383. [CrossRef]

4. Persia, S.; Rea, L. Next generation M2M Cellular Networks: LTE-MTC and NB-IoT capacity analysis for Smart Grids applications. In Proceedings of the 2016 AEIT International Annual Conference (AEIT), Capri, Italy, 5-7 October 2016; pp. 1-6. [CrossRef]

5. Bjornson, E.; Larsson, E.G.; Debbah, M. Massive MIMO for Maximal Spectral Efficiency: How Many Users and Pilots Should Be Allocated? IEEE Trans. Wirel. Commun. 2016, 15, 1293-1308. [CrossRef]

6. Saraereh, O.A.; Khan, I.; Alsafasfeh, Q.; Alemaishat, S.; Kim, S. Low-Complexity Channel Estimation in 5G Massive MIMO-OFDM Systems. Symmetry 2019, 11, 713. [CrossRef]

7. Bjornson, E.; Larsson, E.G.; Marzetta, T.L. Massive MIMO: Ten myths and one critical question. IEEE Commun. Mag. 2016, 54, 114-123. [CrossRef]

8. Vieira, J.; Malkowsky, S.; Nieman, K.; Miers, Z.; Kundargi, N.; Liu, L.; Wong, I.; Öwall, V.; Edfors, O.; Tufvesson, F. A flexible 100-antenna testbed for Massive MIMO. In Proceedings of the 2014 IEEE Globecom Workshops (GC Wkshps), Austin, TX, USA, 8-12 December 2014; pp. 287-293. [CrossRef]

9. Rusek, F.; Persson, D.; Lau, B.K.; Larsson, E.G.; Marzetta, T.L.; Edfors, O.; Tufvesson, F. Scaling Up MIMO: Opportunities and Challenges with Very Large Arrays. IEEE Signal Process. Mag. 2013, 30, 40-60. [CrossRef]

10. Sanguinetti, L.; D'Amico, A.A.; Morelli, M.; Debbah, M. Random Access in Uplink Massive MIMO Systems: How to Exploit Asynchronicity and Excess Antennas. In Proceedings of the 2016 IEEE Global Communications Conference (GLOBECOM), Washington, DC, USA, 4-8 December 2016; pp. 1-5. [CrossRef]

11. Sorensen, J.H.; de Carvalho, E.; Popovski, P. Massive MIMO for crowd scenarios: A solution based on random access. In Proceedings of the 2014 IEEE Globecom Workshops, Austin, TX, USA, 8-12 December 2014; pp. 352-357. [CrossRef]

12. Bjornson, E.; de Carvalho, E.; Larsson, E.G.; Popovski, P. Random access protocol for massive MIMO: Strongest-user collision resolution (SUCR). In Proceedings of the 2016 IEEE International Conference on Communications, Kuala Lumpur, Malaysia, 22-27 May 2016; pp. 1-6. [CrossRef]

13. Study on RAN Improvements for Machine-Type Communications. 3GPP TR 37.868 V11.0.0. September 2011. Available online: https://portal.3gpp.org/desktopmodules/Specifications/SpecificationDetails.aspx? specificationId=2630 (accessed on 20 June 2019).

14. Lien, S.; Chen, K.; Lin, Y. Toward ubiquitous massive accesses in 3GPP machine-to-machine communications. IEEE Commun. Mag. 2011, 49, 66-74. [CrossRef]

15. Ksentini, A.; Hadjadj-Aoul, Y.; Taleb, T. Cellular-based machine-to-machine: Overload control. IEEE Netw. 2012, 26, 54-60. [CrossRef]

16. Duan, S.; Shah-Mansouri, V.; Wang, Z.; Wong, V.W.S. D-ACB: Adaptive Congestion Control Algorithm for Bursty M2M Traffic in LTE Networks. IEEE Trans. Veh. Technol. 2016, 65, 9847-9861. [CrossRef] 
17. Zheng, K.; Ou, S.; Alonso-Zarate, J.; Dohler, M.; Liu, F.; Zhu, H. Challenges of massive access in highly dense LTE-advanced networks with machine-to-machine communications. IEEE Wirel. Commun. 2014, 21, 12-18. [CrossRef]

18. Ali, M.S.; Hossain, E.; Kim, D.I. LTE/LTE-A Random Access for Massive Machine-Type Communications in Smart Cities. IEEE Commun. Mag. 2017, 55, 76-83. [CrossRef]

19. Access Class Barring and Overload Protection. 3GPP TR23.898 V7.0.0. March 2005. Available online: https: / / portal.3gpp.org/desktopmodules/Specifications/SpecificationDetails.aspx?specificationId=977 (accessed on 22 April 2019).

20. He, H.; Du, Q.; Song, H.; Li, W.; Wang, Y.; Ren, P. Traffic-aware ACB scheme for massive access in machine-to-machine networks. In Proceedings of the 2015 IEEE International Conference on Communications, London, UK, 8-12 June 2015; pp. 617-622. [CrossRef]

21. Kapsalis, V.; Hadellis, L.; Karelis, D.; Koubias, S. A dynamic context-aware access control architecture for e-services. Comput. Secur. 2006, 25, 507-521. [CrossRef]

22. Colombo, P.; Ferrari, E. Enhancing NoSQL datastores with fine-grained context-aware access control: A preliminary study on MongoDB. Int. J. Cloud Comput. 2017, 6, 292-305. [CrossRef]

23. Kim, Y.G.; Mon, C.J.; Jeong, D.; Lee, J.O.; Song, C.Y.; Baik, D.K. Context-Aware Access Control Mechanism for Ubiquitous Applications. In Advances in Web Intelligence; Szczepaniak, P.S., Kacprzyk, J., Niewiadomski, A., Eds.; Springer: Berlin/Heidelberg, Germany, 2005; pp. 236-242.

24. Kayes, A.S.M.; Rahayu, W.; Dillon, T.; Chang, E. Accessing Data from Multiple Sources Through Context-Aware Access Control. In Proceedings of the 2018 17th IEEE International Conference on Trust, Security and Privacy in Computing and Communications/12th IEEE International Conference on Big Data Science and Engineering (TrustCom/BigDataSE), New York, NY, USA, 1-3 August 2018; pp. 551-559. [CrossRef]

25. Bjornson, E.; de Carvalho, E.; Sørensen, J.H.; Larsson, E.G.; Popovski, P. A Random Access Protocol for Pilot Allocation in Crowded Massive MIMO Systems. IEEE Trans. Wirel. Commun. 2017, 16, 2220-2234. [CrossRef]

26. Phuyal, U.; Koc, A.T.; Fong, M.H.; Vannithamby, R. Controlling access overload and signaling congestion in M2M networks. In Proceedings of the 2012 Conference Record of the Forty Sixth Asilomar Conference on Signals, Systems and Computers, Pacific Grove, CA, USA, 4-7 November 2012; pp. 591-595. [CrossRef]

27. Ngo, H.Q.; Larsson, E.G.; Marzetta, T.L. Aspects of favorable propagation in massive MIMO. In Proceedings of the 2014 Proceedings of the 22nd European Signal Processing Conference (EUSIPCO), Lisbon, Portugal, 1-5 September 2014; pp. 76-80.

28. Oh, C.; Hwang, D.; Lee, T. Joint Access Control and Resource Allocation for Concurrent and Massive Access of M2M Devices. IEEE Trans. Wirel. Commun. 2015, 14, 4182-4192. [CrossRef]

29. Spatial Channel Model for Multiple Input Multiple Output (MIMO). 3GPP TR 25.996 V13.0.0. December 2015. Available on: https://portal.3gpp.org/desktopmodules/Specifications/SpecificationDetails.aspx? specificationId=1382 (accessed on 7 May 2019).

(C) 2019 by the authors. Licensee MDPI, Basel, Switzerland. This article is an open access article distributed under the terms and conditions of the Creative Commons Attribution (CC BY) license (http://creativecommons.org/licenses/by/4.0/). 